\title{
CONGRUENCE RELATIONS IN DIRECT PRODUCTS
}

\author{
GRANT A. FRASER AND ALFRED HORN ${ }^{1}$
}

ABstract. This paper studies conditions under which every congruence relation $\theta$ in a direct product $A \times B$ of algebras is of the form $\theta_{1} \times \theta_{2}$, where $\theta_{1}$ and $\theta_{2}$ are congruence relations in $A$ and $B$ respectively. It is shown that for any equational class $K$, every such $\theta$ in every $A \times B$ in $K$ has this property if and only if $K$ satisfies certain identities.

It is well known that if $A$ and $B$ are rings with unit element, then every ideal in $A \times B$ is of the form $I_{1} \times I_{2}$, where $I_{1}, I_{2}$ are ideals in $A$ and $B$ respectively. In [1, Problem 40], G. Grätzer asks to characterize those equational classes $K$ of algebras such that for any $A, B \in K$, every congruence relation $\theta$ in $A \times B$ is of the form $\theta_{1} \times \theta_{2}$, where $\theta_{1}, \theta_{2}$ are congruence relations in $A$ and $B$ respectively. ${ }^{2}$ In Theorem 5 below, we shall show that $K$ can be characterized by Malcev-type conditions, that is, by the existence of certain identities in $K$.

We restrict our attention to algebras with finitary operations (possibly infinite in number). The set of congruence relations in $A$ is denoted by $C(A)$. If $\theta \in C(A)$, then $a_{1} \theta a_{2}$ denotes $\left(a_{1}, a_{2}\right) \in \theta$. If $\theta_{1} \in C(A)$ and $\theta_{2} \in C(B)$, let $\theta_{1} \times \theta_{2}$ be the relation $\left\{\left(\left(a_{1}, b_{1}\right),\left(a_{2}, b_{2}\right)\right): a_{1} \theta_{1} a_{2}\right.$ and $\left.b_{1} \theta_{2} b_{2}\right\}$. Clearly $\theta_{1} \times \theta_{2} \in C(A \times B)$. Let $I_{A}=\{(a, a): a \in A\}$ be the identity relation on $A$, and $U_{A}=A \times A$ be the universal relation on $A$. If $a_{1}, a_{2} \in A$, let $\theta\left(a_{1}, a_{2}\right)$ be the smallest congruence relation on $A$ which contains $\left(a_{1}, a_{2}\right)$.

In $C(A \times B)$, we let $\Pi_{1}$ denote the kernel of the projection on $A$. Clearly $\Pi_{1}=\left\{\left(\left(a_{1}, b_{1}\right), \quad\left(a_{2}, b_{2}\right)\right): a_{1}=a_{2}\right\}=I_{A} \times U_{B}$; similarly let $\Pi_{2}=U_{A} \times I_{B}$. It is well known (see [1]) that $C(A)$ is a lattice in which $\theta_{1} \wedge \theta_{2}=\theta_{1} \cap \theta_{2}$, and

$$
\theta_{1} \vee \theta_{2}=\bigcup_{n<\omega}\left(\rho_{0} \circ \rho_{1} \circ \cdots \circ \rho_{n}\right),
$$

where $\rho_{i}=\theta_{1}$ for even $i$, and $\rho_{i}=\theta_{2}$ for odd $i$.

LEMma 1. If $\rho_{1}, \theta_{1} \in C(A)$ and $\rho_{2}, \theta_{2} \in C(B)$, then

$$
\left(\rho_{1} \times \rho_{2}\right) \vee\left(\theta_{1} \times \theta_{2}\right)=\left(\rho_{1} \vee \theta_{1}\right) \times\left(\rho_{2} \vee \theta_{2}\right)
$$

Received by the editors January 15, 1970.

AMS 1969 subject classifications. Primary 0830; Secondary 0245, 0250.

Key words and phrases. Congruence relation, direct product, equational class.

1 This research was supported in part by NSF grant GP-9044.

2 This problem has been solved independently by Tah-Kai $\mathrm{Hu}$. 
Proof. It is obvious that $\rho_{1} \times \rho_{2}$ and $\theta_{1} \times \theta_{2}$ are contained in $\left(\rho_{1} \bigvee \theta_{1}\right) \times\left(\rho_{2} \bigvee \theta_{2}\right)$. Now let $(a, b) \in\left(\rho_{1} \bigvee \theta_{1}\right) \times\left(\rho_{2} \bigvee \theta_{2}\right)$. Then for some $m, a \in \phi_{0} \circ \cdots \circ \phi_{m}$ and $b \in \psi_{0} \circ \cdots \circ \psi_{m}$, where $\phi_{i}=\rho_{1}, \psi_{i}=\rho_{2}$ for even $i$, and $\phi_{i}=\theta_{1}, \psi_{i}=\theta_{2}$ for odd $i$ (since $\phi_{0} \circ \cdots \circ \phi_{n} \subseteq \phi_{0} \circ \cdots \circ \phi_{n+1}$ ); hence

$$
\begin{aligned}
(a, b) \in\left(\phi_{0} \circ \cdots \circ \phi_{m}\right) \times\left(\psi_{0} \circ \cdots \circ \psi_{m}\right) & =\left(\phi_{0} \times \psi_{0}\right) \circ \cdots \circ\left(\phi_{m} \times \psi_{m}\right) \\
& \subseteq\left(\rho_{1} \times \rho_{2}\right) \vee\left(\theta_{1} \times \theta_{2}\right) .
\end{aligned}
$$

Definition. If $\theta \in C(A \times B)$, we say $\theta$ has property $P$ if there exist $\theta_{1} \in C(A), \theta_{2} \in C(B)$ such that $\theta=\theta_{1} \times \theta_{2}$. We say $A \times B$ has property $P$ if every $\theta \in C(A \times B)$ has property $P$. If $K$ is a class of similar algebras, we say $K$ has property $P$ if for all $A, B \in K, A \times B$ has property $P$.

It is easily seen that $A \times B$ has property $P$ if and only if the map $\left(\theta_{1}, \theta_{2}\right) \rightarrow \theta_{1} \times \theta_{2}$ is an isomorphism from $C(A) \times C(B)$ onto $C(A \times B)$.

THEOREM 1. Let $\theta \in C(A \times B)$. Then the following are equivalent:

(1) $\theta$ has property $P$.

(2) $\Pi_{2} \cap\left(\Pi_{1} \bigvee \theta\right) \subseteq \theta$ and $\Pi_{1} \cap\left(\Pi_{2} \vee \theta\right) \subseteq \theta$.

(3) For all $a, a_{1}, a_{2} \in A$ and all $b, b_{1}, b_{2} \in B,\left(a_{1}, b_{1}\right) \theta\left(a_{2}, b_{2}\right)$ implies $\left(a_{1}, b\right) \theta\left(a_{2}, b\right)$ and $\left(a, b_{1}\right) \theta\left(a, b_{2}\right)$.

Proof. (1) $\rightarrow(2):$ If $\theta=\theta_{1} \times \theta_{2}$ then by Lemma 1 ,

$$
\begin{aligned}
\Pi_{2} \cap\left(\Pi_{1} \vee \theta\right) & =\Pi_{2} \cap\left(\left(I_{A} \times U_{B}\right) \vee\left(\theta_{1} \times \theta_{2}\right)\right) \\
& =\left(U_{A} \times I_{B}\right) \cap\left(\theta_{1} \times U_{B}\right)=\theta_{1} \times I_{B} \subseteq \theta,
\end{aligned}
$$

and similarly $\Pi_{1} \cap\left(\Pi_{2} \vee \theta\right) \subseteq \theta$.

$(2) \rightarrow(3)$ : Suppose $\left(a_{1}, b_{1}\right) \theta\left(a_{2}, b_{2}\right)$. Since $\left(a_{1}, b\right) \Pi_{1}\left(a_{1}, b_{1}\right)$ and $\left(a_{2}, b_{2}\right) \Pi_{1}\left(a_{2}, b\right)$ we have $\left(a_{1}, b\right)\left(\Pi_{1} \circ \theta \circ \Pi_{1}\right)\left(a_{2}, b\right)$. Since $\Pi_{1} \circ \theta \circ \Pi_{1}$ $\subseteq \theta \vee \Pi_{1}$, it follows that

$$
\left(a_{1}, b\right)\left(\Pi_{2} \cap\left(\theta \vee \Pi_{1}\right)\right)\left(a_{2}, b\right)
$$

and by $(2),\left(a_{1}, b\right) \theta\left(a_{2}, b\right)$. Similarly $\left(a, b_{1}\right) \theta\left(a, b_{2}\right)$.

$(3) \rightarrow(1):$ Let

$$
\begin{aligned}
& \theta_{1}=\left\{\left(a_{1}, a_{2}\right): \text { for some } b,\left(a_{1}, b\right) \theta\left(a_{2}, b\right)\right\}, \\
& \theta_{2}=\left\{\left(b_{1}, b_{2}\right) \text { : for some } a,\left(a, b_{1}\right) \theta\left(a, b_{2}\right)\right\} .
\end{aligned}
$$

Then by (3), $a_{1} \theta_{1} a_{2}$ implies $\left(a_{1}, b\right) \theta\left(a_{2}, b\right)$ for all $b \in B$. It is very easy to verify that $\theta_{1} \in C(A), \theta_{2} \in C(B)$ and $\theta=\theta_{1} \times \theta_{2}$.

Corollary 1. If $C(A \times B)$ is a distributive lattice, then $A \times B$ has property $P$. 
Proof. Since $\Pi_{1} \cap \Pi_{2}=I_{A \times B}$, it is easily seen that (2) holds.

Corollary 1 was pointed out to us by Alfred Hales. He gave a different proof of the sufficiency part of the next theorem, from which Corollary 1 also follows.

Theorem 2. If $\theta \in C(A \times B)$, then $\theta$ has property $P$ if and only if

$$
\left(\Pi_{1} \vee \theta\right) \cap\left(\Pi_{2} \vee \theta\right)=\theta \text {. }
$$

Proof. If $\theta=\theta_{1} \times \theta_{2}$, then by Lemma 1 ,

$$
\begin{aligned}
\left(\Pi_{1} \vee \theta\right) & \cap\left(\Pi_{2} \vee \theta\right) \\
= & {\left[\left(I_{A} \times U_{B}\right) \vee\left(\theta_{1} \times \theta_{2}\right)\right] \cap\left[\left(U_{A} \times I_{B}\right) \vee\left(\theta_{1} \times \theta_{2}\right)\right] } \\
& =\left[\left(I_{A} \vee \theta_{1}\right) \times\left(U_{B} \vee \theta_{2}\right)\right] \cap\left[\left(U_{A} \vee \theta_{1}\right) \times\left(I_{B} \vee \theta_{2}\right)\right] \\
& =\left(\theta_{1} \times U_{B}\right) \cap\left(U_{A} \times \theta_{2}\right)=\theta_{1} \times \theta_{2}=\theta .
\end{aligned}
$$

Conversely, if (4) holds, then (2) is an immediate consequence.

Theorem 3. Let $A$ and $B$ be similar algebras. Then the following are equivalent:

(5) $A \times B$ has property $P$.

(6) For all $a_{1}, a_{2} \in A$ and all $b_{1}, b_{2} \in B, \theta\left(a_{1}, a_{2}\right) \times \theta\left(b_{1}, b_{2}\right)$ $=\theta\left(\left(a_{1}, b_{1}\right),\left(a_{2}, b_{2}\right)\right)$.

(7) For all $a_{1}, a_{2}, a \in A$ and all $b_{1}, b_{2}, b \in B,\left(\left(a_{1}, b\right),\left(a_{2}, b\right)\right)$ and $\left(\left(a, b_{1}\right),\left(a, b_{2}\right)\right)$ are in $\theta\left(\left(a_{1}, b_{1}\right),\left(a_{2}, b_{2}\right)\right)$.

Proof. (5) $\rightarrow(6)$ : Clearly, $\left(\left(a_{1}, b_{1}\right),\left(a_{2}, b_{2}\right)\right) \in \theta\left(a_{1}, a_{2}\right) \times \theta\left(b_{1}, b_{2}\right)$. Suppose $\left(\left(a_{1}, b_{1}\right),\left(a_{2}, b_{2}\right)\right) \in \theta \in C(A \times B)$. By $(5), \theta=\theta_{1} \times \theta_{2}$ for some $\theta_{1} \in C(A), \theta_{2} \in C(B)$. Therefore $a_{1} \theta_{1} a_{2}$ and $b_{1} \theta_{2} b_{2}$. Hence $\theta=\theta_{1} \times \theta_{2}$ $\supseteq \theta\left(a_{1}, a_{2}\right) \times \theta\left(b_{1}, b_{2}\right)$. This proves (6).

$(6) \rightarrow(7):$ We have $\left(\left(a_{1}, b\right),\left(a_{2}, b\right)\right) \in \theta\left(a_{1}, a_{2}\right) \times \theta\left(b_{1}, b_{2}\right)=\theta\left(\left(a_{1}, b_{1}\right),\left(a_{2}, b_{2}\right)\right)$ and similarly $\left(\left(a, b_{1}\right),\left(a, b_{2}\right)\right) \in \theta\left(\left(a_{1}, b_{1}\right),\left(a_{2}, b_{2}\right)\right)$.

$(7) \rightarrow(5)$ : We show that (3) holds for every $\theta \in C(A \times B)$. Suppose $\left(a_{1}, b_{1}\right) \theta\left(a_{2}, b_{2}\right)$. Then $\theta \supseteq \theta\left(\left(a_{1}, b_{1}\right),\left(a_{2}, b_{2}\right)\right)$. Therefore by $(7)$, (3) follows.

TheOREM 4. Let $K$ be any class of similar algebras. Then $K$ has property $P$ if and only if

(8) for all $A, B \in K$, all $a_{1}, a_{2} \in A$, and all $b_{1}, b_{2}, b \in B$,

$$
\left(\left(a_{1}, b\right),\left(a_{2}, b\right)\right) \in \theta\left(\left(a_{1}, b_{1}\right),\left(a_{2}, b_{2}\right)\right) .
$$

Proof. The necessity of (8) is obvious by (7). To prove the sufficiency, we show that (7) holds for all $A, B \in K$. Let $a_{1}, a_{2}, a \in A$ and $b_{1}, b_{2}, b \in B$. Then by (8) 


$$
\left(\left(a_{1}, b\right),\left(a_{2}, b\right)\right) \in \theta\left(\left(a_{1}, b_{1}\right),\left(a_{2}, b_{2}\right)\right) .
$$

Applying (8) to $B \times A$, we have

$$
\left(\left(b_{1}, a\right),\left(b_{2}, a\right)\right) \in \theta\left(\left(b_{1}, a_{1}\right),\left(b_{2}, a_{2}\right)\right) .
$$

Using the canonical isomorphism of $B \times A$ with $A \times B$, we have

$$
\left(\left(a, b_{1}\right),\left(a, b_{2}\right)\right) \in \theta\left(\left(a_{1}, b_{1}\right),\left(a_{2}, b_{2}\right)\right) .
$$

Corollary 2. If $K$ is an equational class such that for every $A \in K$ with two generators and for every $B \in K$ with three generators, $A \times B$ has property $P$, then $K$ has property $P$.

LEMma 2. If $u, v, c_{0}, c_{1} \in A$, then $(u, v) \in \theta\left(c_{0}, c_{1}\right)$ if and only if for some $m \geqq 1, n \geqq 1$, there exist $(m+1)$-ary polynomials $p_{1}, \cdots, p_{n}$, elements $z_{i j}$ of $A$ for $1 \leqq i \leqq n, 1 \leqq j \leqq m$, and integers $k(1), \cdots, k(n)$ such that

$$
\begin{array}{r}
k(i)=0 \text { or } 1 \quad \text { for } 1 \leqq i \leqq n, \\
u=p_{1}\left(c_{k(1)}, z_{11}, \cdots, z_{1 m}\right), \quad v=p_{n}\left(c_{1-k(n)}, z_{n 1}, \cdots, z_{n m}\right), \\
p_{i}\left(c_{1-k(i)}, z_{i 1}, \cdots, z_{i m}\right)=p_{i+1}\left(c_{k(i+1)}, z_{i+1,1}, \cdots, z_{i+1, m}\right) \\
\quad \text { for } 1 \leqq i \leqq n-1 .
\end{array}
$$

PROOF. This is a paraphrase of Theorem 3, p. 54 of [1]. As pointed out by G. Grätzer, we may take $m=n$ and $k(i)=0$ for even $i$ and $k(i)=1$ for odd $i$.

TheOREM 5. Let $K$ be an equational class of algebras. Then $K$ has property $P$ if and only if for some $n \geqq 1$ and some $m \geqq 1$, there exist $(m+1)$-ary polynomials $p_{1}, \cdots, p_{n}$, binary polynomials $q_{i j}\left(x_{0}, x_{1}\right)$ and ternary polynomials $r_{i j}\left(x_{0}, x_{1}, x_{2}\right)$ for $1 \leqq i \leqq n, 1 \leqq j \leqq m$, and integers $k(1), \cdots, k(n)$ which are 0 or 1 such that the following identities hold in all members of $K$ :

$$
\begin{gathered}
x_{0}=p_{1}\left(x_{k(1)}, q_{11}, \cdots, q_{1 m}\right), \quad x_{1}=p_{n}\left(x_{1-k(n)}, q_{n 1}, \cdots, q_{n m}\right), \\
p_{i}\left(x_{1-k(i)}, q_{i 1}, \cdots, q_{i m}\right)=p_{i+1}\left(x_{k(i+1)}, q_{i+1,1}, \cdots, q_{i+1, m}\right),
\end{gathered}
$$

$$
1 \leqq i \leqq n-1 \text {, }
$$

$$
\begin{gathered}
x_{2}=p_{1}\left(x_{k(1)}, r_{11}, \cdots, r_{1 m}\right)=p_{n}\left(x_{1-k(n)}, r_{n 1}, \cdots, r_{n m}\right), \\
p_{i}\left(x_{1-k(i)}, r_{i 1}, \cdots, r_{i m}\right)=p_{i+1}\left(x_{k(i+1)}, r_{i+1,1}, \cdots, r_{i+1, m}\right),
\end{gathered}
$$

$$
1 \leqq i \leqq n-1 \text {. }
$$

Proof. Suppose $K$ has property $P$. Let $A$ be the free $K$ algebra with two free generators $x_{0}, x_{1}$ and $B$ be the free $K$ algebra with three free generators $x_{0}, x_{1}, x_{2}$. Then by Theorem $4,\left(\left(x_{0}, x_{2}\right),\left(x_{1}, x_{2}\right)\right)$ 
$\in \theta\left(\left(x_{0}, x_{0}\right),\left(x_{1}, x_{1}\right)\right)$. To obtain (9), we use Lemma 2, the fact that every element of $A \times B$ is of the form $\left(q\left(x_{0}, x_{1}\right), r\left(x_{0}, x_{1}, x_{2}\right)\right)$, and that $p\left(\left(u_{0}, v_{0}\right), \cdots,\left(u_{m}, v_{m}\right)\right)=\left(p\left(u_{0}, \cdots, u_{m}\right), p\left(v_{0}, \cdots, v_{m}\right)\right)$ for every $(m+1)$-ary polynomial. Conversely, if we assume (9), then by substituting $x_{0}=a_{0}, x_{1}=a_{1}$ in the first three lines of (9) and $x_{0}=b_{0}, x_{1}=b_{1}$ and $x_{2}=b$ in the last two lines of (9), we see by Lemma 2 that (8) holds.

We close with some examples and remarks. As pointed out before, the class $K_{R}$ of all rings with unit element has property $P$. More generally, if $K$ is such that there exist binary polynomials + and $\cdot$, and constants (or polynomials which are constant in $K$ ) 0 and 1 such that the identities $x \cdot 1=x+0=0+x=x$ and $x \cdot 0=0$ hold in $K$, then $K$ has property $P$. This follows from Theorem 5 with $n=1, m=2$, $p_{1}(x, y, z)=x \cdot y+z, q_{11}=1, q_{12}=0, r_{11}=0, r_{12}=x_{2}$ and $k(1)=0$.

Another example is the class $K_{L}$ of all lattices. In this case, the condition of Theorem 5 holds with $n=1, m=2, p_{1}(x, y, z)=(x \wedge y) \vee z$, $q_{11}=x_{0} \vee x_{1}, q_{12}=x_{0} \wedge x_{1}, r_{11}=x_{2}, r_{12}=x_{2}$ and $k(1)=0$. The fact that $K_{L}$ has property $P$ also follows from Corollary 1 . Property $P$ also extends to any class $K$ (such as the class of lattice ordered groups) of algebras each of which is a lattice under some of its operations. There exist equational classes $K$ having property $P$ but such that $C(A \times B)$ is not distributive for some $A, B \in K$. As an example, let $K=K_{R}$ and $A=B=$ the ring of all polynomials in $x, y$ over the rationals. If $(p)$ denotes the principal ideal generated by $p$, then in $C(A)$, we have

$$
(x+y) \cap((x) \vee(y)) \Phi((x+y) \cap(x)) \vee((x+y) \cap(y)) .
$$

We may define property $P$ in the obvious way for arbitrary direct products. However, the following theorem shows that property $P$ can only hold for essentially finite direct products.

Theorem 6. If $A=\prod_{i \in I} A_{i}$ has property $P$, then $A_{i}$ has one element for all but a finite number of $i$.

Proof. Let $\theta=\{(x, y): x(i)=y(i)$ for almost all $i\}$. Then $\theta \in C(A)$. If $\theta=\prod_{i \in I} \theta_{i}$, then $\theta_{i}$ must be $U_{A_{i}}$ for all $i$, since $x \theta y$ whenever $x(j)=y(j)$ for all $j \in I, j \neq i$. Therefore $\theta=U_{A}$, from which it follows that $A_{i}$ is trivial for almost all $i$.

\section{REFERENCES}

1. G. Grätzer, Universal algebra, University Series in Higher Math., Van Nosstrand, Princeton, N. J., 1968.

University of California, los Angeles, California 90024 